\title{
An open-label, single-arm pilot study of EUS-guided brachytherapy with phosphorus-32 microparticles in combination with gemcitabine +/- nab-paclitaxel in unresectable locally advanced pancreatic cancer (OncoPaC-1): Technical details and study protocol
}

Manoop S. Bhutani ${ }^{1}$, Jason B. Klapman ${ }^{2}$, Richard Tuli³, Ghassan El-Haddad4, Sarah Hoffe ${ }^{5}$, Franklin C. L. Wong ${ }^{6}$, Beth Chasen ${ }^{6}$, David R. Fogelman7, Simon K. Lo ${ }^{8}$, Nicholas N. Nissen ${ }^{9}$, Andrew E. Hendifar ${ }^{10}$, Gauri Varadhachary7, Matthew H. G. Katz ${ }^{11}$, William D. Erwin ${ }^{12}$, Eugene J. Koay ${ }^{13}$, Eric P. Tamm ${ }^{14}$, Ben S. Singh ${ }^{1}$, Rutika Mehta ${ }^{2}$, Robert A. Wolff7, Ashish Somann ${ }^{15}$, Irina M. Cazacu ${ }^{1}$, Joseph M. Herman ${ }^{13}$ ${ }^{1}$ Departments of Gastroenterology, Hepatology and Nutrition, ${ }^{6}$ Nuclear Medicine, ${ }^{7}$ Gastrointestinal Medical Oncology, ${ }^{11}$ Surgical Oncology, ${ }^{12}$ Imaging Physics, ${ }^{13}$ Radiation Oncology and ${ }^{14}$ Diagnostic Radiology, The University of Texas MD Anderson Cancer Center, Houston, TX, Departments of ${ }^{2}$ Gastrointestinal Oncology and ${ }^{4}$ Diagnostic Imaging and Interventional Radiology, ${ }^{5}$ Radiation Oncology, Moffitt Cancer Center, Tampa, FL, ${ }^{3}$ Department of Radiation Oncology, Memorial Sloan Kettering Cancer Center, New York, NY, ${ }^{8}$ Division of Digestive and Liver Diseases, Cedars Sinai Medical Center, Departments of ${ }^{9}$ Surgery and ${ }^{10} \mathrm{Hematology/Oncology,} \mathrm{Cedars} \mathrm{Sinai} \mathrm{Medical} \mathrm{Center,} \mathrm{Los} \mathrm{Angeles,} \mathrm{CA,} \mathrm{USA;}$ ${ }^{15}$ OncoSil Medical Ltd., Sydney, Australia

\begin{abstract}
Current treatment options for patients with unresectable locally advanced pancreatic cancer (LAPC) include chemotherapy alone or followed by chemoradiation or stereotactic body radiotherapy. However, the prognosis for these patients remains poor, with a median overall survival $<12$ months. Therefore, novel treatment options are needed. Currently, there is no brachytherapy device approved for pancreatic cancer treatment. Hereby, we present the protocol of a prospective, multicenter, interventional, open-label, single-arm pilot study (OncoPac-1, Clinicaltrial.gov-NCT03076216) aiming to determine the safety and efficacy of Phosphorus-32 when implanted directly into pancreatic tumors using EUS guidance, for patients with unresectable LAPC undergoing chemotherapy (gemcitabine \pm nab-paclitaxel).
\end{abstract}

Key words: Brachytherapy, chemotherapy, endoscopy, EUS, fine needle injection, intratumoral therapy, neoplasia, pancreatic cancer, phosphorus-32, radiotherapy

\begin{tabular}{|l|l|}
\hline \multicolumn{2}{|c|}{ Access this article online } \\
\hline Quick Response Code: & Website: \\
\hline & www.eusjournal.com \\
\cline { 2 - 2 } & DOI: \\
\hline
\end{tabular}

This is an open access journal, and articles are distributed under the terms of the Creative Commons Attribution-NonCommercial-ShareAlike 4.0 License, which allows others to remix, tweak, and build upon the work non-commercially, as long as appropriate credit is given and the new creations are licensed under the identical terms.

For reprints contact: reprints@medknow.com

How to cite this article: Bhutani MS, Klapman JB, Tuli R, El-Haddad G, Hoffe S, Wong FC, et al. An open-label, single-arm pilot study of EUSguided brachytherapy with phosphorus-32 microparticles in combination with gemcitabine $+/$ - nab-paclitaxel in unresectable locally advanced pancreatic cancer (OncoPaC-1): Technical details and study protocol. Endosc Ultrasound 2020;9:24-30.

\section{Address for correspondence}

Dr. Manoop S. Bhutani, Department of Gastroenterology, Hepatology and Nutrition, The University of Texas Anderson Cancer Center, 1515 Holcombe Blvd., Houston 77030-4009, Texas, USA. E-mail: manoop.bhutani@mdanderson.org Dr. Joseph M. Herman, Department of Radiation Oncology, The University of Texas MD Anderson Cancer Center, T. Boone Pickens Academic Tower (FCT6.6080), 1515 Holcombe Blvd., Unit 1442, Houston, Texas 77030, USA. E-mail: jmherman@mdanderson.org Received: 2019-04-23; Accepted: 2019-06-24; Published online: 2019-10-30 


\section{INTRODUCTION}

Pancreatic ductal adenocarcinoma is associated with a poor prognosis and has an increasing impact on cancer-related mortality worldwide. Pancreatic cancer is projected to become the second leading cause of cancer-related deaths by $2030 .^{[1]}$

There is no uniform consensus regarding standard of care treatment of unresectable locally advanced pancreatic cancer (LAPC). The current treatment options include chemotherapy alone or chemotherapy followed by chemoradiotherapy or stereotactic body radiation therapy (SBRT). Conventional radiotherapy (CRT) over 5-6 weeks has been used to treat patients with advanced disease and is usually given concurrently with gemcitabine or fluoropyrimidine-based chemotherapy. However, CRT is limited by the amount of radiation that can be delivered to the gastrointestinal (GI) tract due to side effects. ${ }^{[2]}$ SBRT uses high doses of RT that can be delivered in only 1 week and offers a similar local control as CRT while sparing surrounding normal tissues. ${ }^{[3]}$ While these advances have led to improved tumor control, downstaging remains limited and thus the likelihood of surgery is low. In addition, the current doses of radiation have resulted in pathological complete remission (pCR) rates of only $10 \%$, suggesting that higher radiotherapy doses are needed for achieving better pCR rates. ${ }^{[4]}$

Brachytherapy is a form of radiotherapy where radioactive seeds, microparticles, or liquids are placed directly into or adjacent to the tumor. This technique is currently used in the treatment of prostate, cervical, and breast cancer, but also in other malignancies (lung, head and neck, and liver cancer). ${ }^{[5-8]}$ With brachytherapy, the target tissue is exposed to a steady emission of low-energy gamma, X-rays, or beta particles, which leads to localized tissue injury and tumor ablation. Brachytherapy can deliver a much higher dose of radiation to the tumor when compared to external beam radiation therapy where radiation beams need to traverse normal structures to treat the tumor, thus resulting in collateral toxicity and damage to normal tissue.

There is currently no brachytherapy device approved for the treatment of pancreatic cancer. However, there is evidence to suggest that brachytherapy can deliver a higher dose of radiation and has the potential to offer local control and to provide palliative benefits in patients with LAPC who have few treatment options and whose prognosis is otherwise poor. ${ }^{[9-13]}$ Trials and commercialization of brachytherapy treatments and devices utilizing radioisotopes of phosphorus-32 (P-32), iodine, gold, iridium, and yttrium have been undertaken. ${ }^{[13-18]}$ However, none of these radioactive agents has entered routine clinical practice.

Various techniques for delivery of local radiation have been reported. Initially, intraoperative brachytherapy was used for patients who received palliative surgery. ${ }^{[18]}$ Other methods to place radioactive seeds within the tumor include computed tomography (CT) or ultrasound-guided procedures. ${ }^{[19]}$ EUS has evolved as an interventional procedure, and multiple agents can be delivered under EUS guidance. ${ }^{[20]}$ Thus, EUS is not only useful in the delivery of fiducial markers for guiding external radiotherapy but can also be employed to deliver radioactive seeds into solid tumors. Compared with percutaneous implantation using CT or abdominal ultrasound, EUS provides a clear real-time image, with a more direct puncture pathway.

Conversion to resectability is one of the goals of the treatment in LAPC, although actual downstaging of tumors with the current treatment strategies is very uncommon. EUS-guided brachytherapy in conjunction with chemotherapy may increase the proportion of LAPC patients undergoing surgery or result in more durable local control than conventional treatments.

\section{RATIONALE AND OBJECTIVE}

P-32 has been previously investigated in combination with gemcitabine monotherapy in 23 patients with LAPC and metastatic disease in two phase II clinical studies which demonstrated acceptable tolerability and safety profile. One of the studies ${ }^{[21]}$ was presented as an abstract and the other one is on file with the company. Efficacy data showed potential with evidence of a target tumor response rate of $23 \%$ and a target disease control rate of $82 \%$. These studies suggested that EUS-guided implantation of P-32 is a feasible method of delivery.

The OncoPac-1 multicenter clinical trial is further investigating the safety of P-32 radiolabeled microparticles (OncoSil $\left.{ }^{\mathrm{TM}}\right)$ when implanted directly into pancreatic tumors using EUS for patients with unresectable LAPC undergoing 
chemotherapy (clinicaltrials.gov, NCT03076216). This is the first US experience with P-32. The implanted activity is intended to deliver an average radiation absorbed dose of $100 \mathrm{~Gy}( \pm 20 \%)$ within the tumor. The dose of $100 \mathrm{~Gy}$ proposed in the present OncoPac-1 study has been selected as it has shown to be safe and well tolerated in a previous phase II pancreatic cancer clinical trial (DB2-201) that encompassed the largest pool of patients to date (17 patients). ${ }^{[21]}$

In the OncoPac-1 trial, the eligible patients receive either gemcitabine or gemcitabine + nab-paclitaxel chemotherapy plus P-32 brachytherapy.

The primary endpoint is safety and tolerability of P-32 administered in combination with chemotherapy. The common terminology criteria for adverse events (AEs) are being used to grade and report AEs. The following secondary endpoints are also being assessed: feasibility, local progression-free survival, progression-free survival, overall survival, pain scores, impaired function, and volumetric target tumor changes [Table 1].

\section{STUDY DESIGN}

OncoPac-1 is a prospective, multicenter, interventional, open-label, single-arm pilot study aiming to determine the safety of P-32 when implanted directly into pancreatic tumors using EUS guidance, for patients with unresectable LAPC undergoing chemotherapy (gemcitabine \pm nab-paclitaxel) [Figure 1]. This clinical trial is being conducted at 3 sites in the United States.

Patients are enrolled into the study only if they meet all of the required criteria at screening. The key eligibility criteria for this study are as follows: histological-/ cytological-proven pancreatic adenocarcinoma; unresectable LAPC, without distant metastasis; target tumor diameter 2-6 cm, Eastern Cooperative Oncology Group performance Status 0-1; and naïve treatment patients [Table 2].

The appropriate chemotherapy regimen is determined by the treating oncologist on an individual subject basis and administered in accordance with the manufacturer's approved intended purpose, indications for use, and conditions of approval. Chemotherapy commences at a maximum of 14 days postenrollment. The EUS-guided implantation of P-32
Table 1. Study endpoints

\begin{tabular}{|c|c|}
\hline \multicolumn{2}{|c|}{ Study endpoints for OncoPac-1 } \\
\hline \multicolumn{2}{|l|}{ Primary endpoint } \\
\hline $\begin{array}{l}\text { Safety and } \\
\text { tolerability }\end{array}$ & $\begin{array}{l}\text { Adverse events are graded and } \\
\text { reported using The CTCAE version } 4.0\end{array}$ \\
\hline \multicolumn{2}{|l|}{ Secondary endpoints } \\
\hline $\begin{array}{l}\text { Local } \\
\text { progression-free } \\
\text { survival }\end{array}$ & $\begin{array}{l}\text { Time from enrollment to the date } \\
\text { of the CT scan that shows local } \\
\text { tumor progression or date of death. } \\
\text { Individuals are assessed for target } \\
\text { tumor response every } 8 \text { weeks } \\
\text { from visit } 1 \text { until end of study }\end{array}$ \\
\hline $\begin{array}{l}\text { Progression-free } \\
\text { survival }\end{array}$ & $\begin{array}{l}\text { Time from enrollment to the } \\
\text { date of tumor progression or of } \\
\text { recurrence. Progression of disease } \\
\text { outside of the pancreas are } \\
\text { measured by the investigator in } \\
\text { accordance to RECIST } 1.1 \text { criteria }\end{array}$ \\
\hline Overall survival & $\begin{array}{l}\text { Time from enrollment to the } \\
\text { date of death from any cause }\end{array}$ \\
\hline Pain scores & $\begin{array}{l}\text { Pain is assessed using the EORTC QLQ- } \\
\text { PAN26 assessment questionnaire and } \\
\text { a numerical rating scale. Measured } \\
\text { at each study visit for the duration of } \\
\text { the study, an average of } 12 \text { months }\end{array}$ \\
\hline Impaired function & $\begin{array}{l}\text { Impaired function is evaluated using } \\
\text { ECOG performance status and Karnofsky } \\
\text { performance status. Measured at } \\
\text { each study visit for the duration of } \\
\text { the study, an average of } 12 \text { months }\end{array}$ \\
\hline $\begin{array}{l}\text { Target tumor } \\
\text { volumetric change }\end{array}$ & $\begin{array}{l}\text { Tumor response is evaluated looking } \\
\text { at the change in target tumor volume } \\
\text { measurement. A central reading center } \\
\text { (ImagelQ) will analyze all CT scans to } \\
\text { measure target tumor volume changes } \\
\text { from baseline. The baseline tumor } \\
\text { volume measurement will also be used to } \\
\text { determine the P- } 32 \text { volume to be injected }\end{array}$ \\
\hline \multicolumn{2}{|c|}{$\begin{array}{l}\text { CTCAE: Common Terminology Criteria for Adverse Events, CT: Computed } \\
\text { tomography, ECOG: Eastern Cooperative Oncology Group, } \\
\text { EORTC QLQ-PAN26: European Organization for Research and Treatment of } \\
\text { Cancer Pancreatic Cancer module }\end{array}$} \\
\hline
\end{tabular}

microparticles occurs during the $4^{\text {th }}$ or $5^{\text {th }}$ week of the $1^{\text {st }}$ cycle of chemotherapy. Following implantation, the prescribed chemotherapy regimen is resumed. Due to the potential of chemotherapy agents to be radiosensitizing, at least $48 \mathrm{~h}$ must elapse either side of the P-32 implantations to the start/finish of the chemotherapy infusion.

To assess the intratumoral and potential extratumoral distribution of microparticles, bremsstrahlung whole-body planar plus single-bed single photon emission CT/(SPECT)/CT imaging is performed within $4 \mathrm{~h}$ postimplantation and repeated 7 days after.

Follow-up CT scans are repeated every 8 weeks to assess volumetric tumor response. A medical review of all AEs reported by patients will continue until 104 weeks post enrollment of the last patient. 


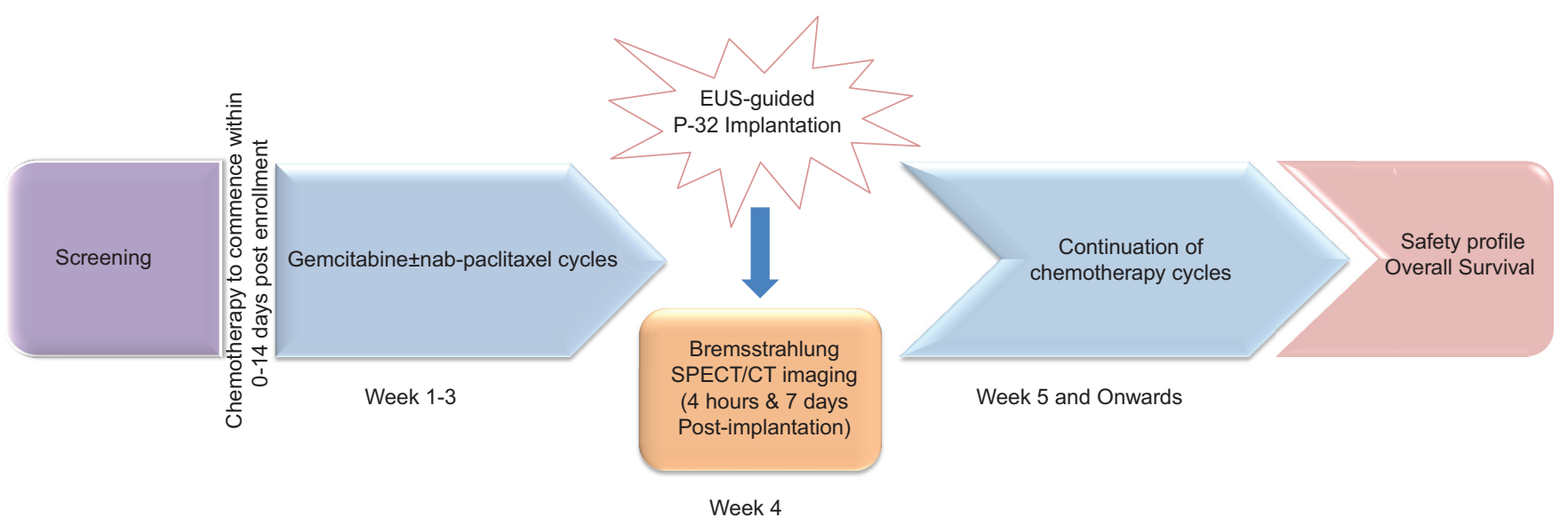

Figure 1. Study design

\section{METHODS}

\section{Study device}

OncoSil is an investigational brachytherapy device containing P-32-radiolabeled microparticles uniformly suspended within a diluent. The P-32 microparticles have been designed to deliver a localized distribution of beta-radiation within the target tumor. The radiation from these particles causes direct damage to cancer cell DNA, which renders them incapable of further cell division and proliferation. Through this mechanism, P-32 is able to stop cancer cells from multiplying which leads to tumor shrinkage. The diluent is a viscous suspension that serves as a carrier to facilitate intratumoral implantation of the microparticles.

The microparticles are manufactured in Germany, and P-32 is produced via neutron activation of the microparticles in a nuclear reactor. The activity for each patient is dispensed, sterilized, and packaged for shipment to the study site. Twenty-eight days between placing an order and availability of P-32 at the study site is required.

\section{Dose calculation and preparation}

The baseline target tumor volume is estimated from the screening/baseline CT scan and is used to calculate the volume of diluent containing the P-32 to be implanted. The P-32 microparticles and diluent are combined in accordance with a predefined suspension preparation protocol.

A single dose is implanted into the tumor using the final suspension of $\mathrm{P}-32$ with a radioactivity concentration of $6.6 \mathrm{MBq} / \mathrm{mL}$ (the radioactivity concentration required, with an implanted
Table 2. Inclusion and exclusion criteria for OncoPac-1 enrollment

Key eligibility criteria

Histologically or cytologically proven pancreatic adenocarcinoma Unresectable locally advanced pancreatic carcinoma

Pancreatic target tumor diameter: $2-6 \mathrm{~cm}$

ECOG performance status $0-1$

No distant metastases

No prior radiotherapy or chemotherapy for pancreatic cancer $\geq 18$ years of age at screening

ECOG: Eastern Cooperative Oncology Group

volume/tumor volume of $8 \%$, to deliver $100 \mathrm{~Gy}$ to the tumor mass).

The two equations shown below are used to calculate actual volume of P-32 dispensed and then implanted:

Volume to be implanted $(\mathrm{mL})$

$$
=\text { Tumor volume }(\mathrm{mL}) \times \frac{8}{100}
$$

Activity to be implanted $(\mathrm{MBq})=$ Volume to be implanted $(\mathrm{mL}) \times 6.6$

The required volume is drawn up into a 3 or $5 \mathrm{~mL}$ syringe within a beta shield which is placed in a lead transport box. The dispensed dose must be used within $24 \mathrm{~h}$ of preparation and stored between $15^{\circ} \mathrm{C}$ and $25^{\circ} \mathrm{C}$. The nuclear medicine technologist or a radiopharmacist dispenses the dose and transports it to the endoscopy suite.

\section{Preimplantation considerations}

An overview of the P-32 implantation is shown in Figure 2. 


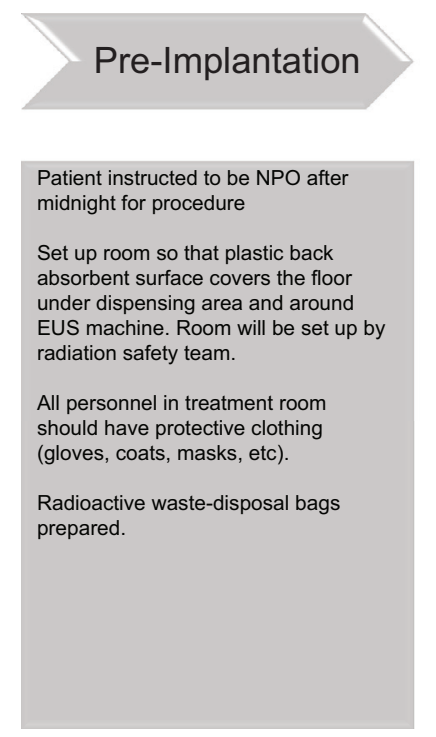

Figure 2. Overview of phosphorus-32 implantation
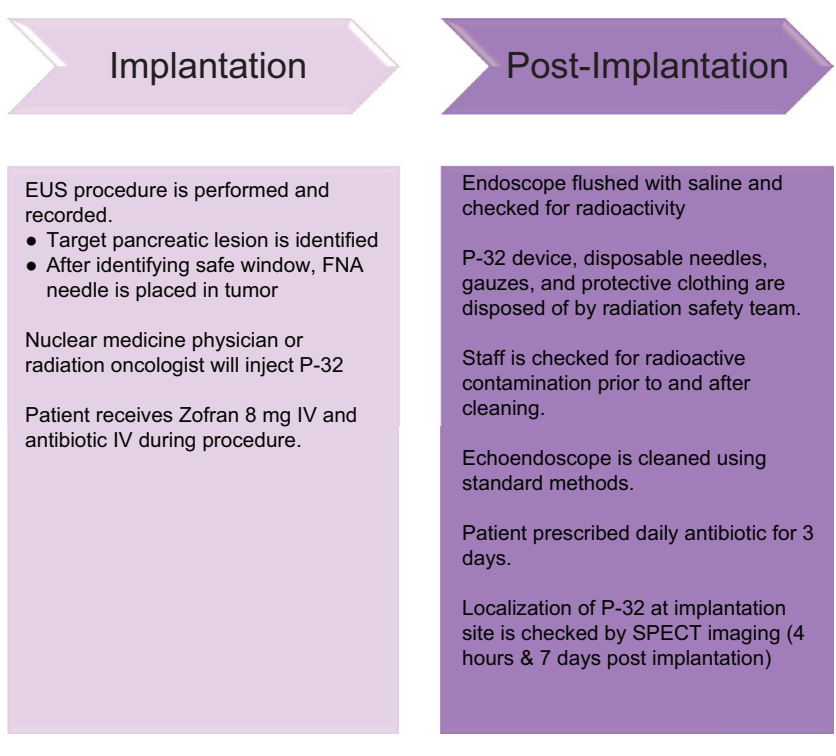

Radiation safety is an important consideration in the use of P-32 brachytherapy, from the receipt of the radioactive material, its preparation and administration to the patient, to the release of the patient from the treatment facility. The beta emissions from P-32 can present an inhalation, skin, and eye dose hazard; thus, appropriate personnel protective equipment must be used. A radiation monitor suitable for detecting P-32 is used to assess operational areas for contaminations. A special decontamination kit must be available for immediate use in case of any spills of P-32.

The endoscopy suite is carefully prepared before the procedure. Spill tray lined with plastic-backed absorbent material is used to cover the floor. The room is equipped with clinical radioactive waste disposal bags, protective clothing, monitor suitable for detecting P-32, radiation spill kit, and appropriate personnel dosimetry for handling P-32.

\section{Implantation procedure}

Periprocedural intravenous prophylactic antibiotic treatment is administered (on the day of the procedure and for 2 days after).

The target pancreatic lesion is identified using EUS. A 22 gauge FNA needle is loaded through the biopsy channel of the echoendoscope and slowly advanced into the target pancreatic tumor. Once the FNA needle is in a satisfactory position within the tumor and safe insertion window is identified, the stylet is removed and the syringe with P-32 is attached to the FNA needle.
P-32 is injected in the center of the tumor using a needle tracking technique by pulling the needle back when the injection becomes difficult. A radiation oncologist or a nuclear medicine physician slowly depresses the plunger of the syringe in beta shield to implant P-32. The injection is seen as an echogenic blush within the tumor [Figure 3]. Saline is used to flush the P-32 remaining in the needle into the tumor, and then, the needle is withdrawn back into the sheath. Additional flush with saline is done with the sheath within the GI lumen. The sheath is not fully retracted into the echoendoscope to decrease the risk of scope contamination. The echoendoscope is gently withdrawn out of the patient.

\section{Postimplantation considerations}

Following the procedure, all materials are sterilized and disposed of following radiation safety guidelines and under the supervision of the institutional Radiation Safety Department.

After completion of the implantation, the syringe containing any residual P-32 suspension is recapped, placed into the lead transport box, and transferred to the Nuclear Medicine Department/Radiopharmacy for measurement and then decay.

The scope tip is held over a clinical waste bag and rinsed with $50 \mathrm{~mL}$ water. The echoendoscope and the staff are checked for any radioactive contamination using the radiation monitor. The echoendoscope is washed and sterilized using the standard methods for cleaning the equipment at the study site. 


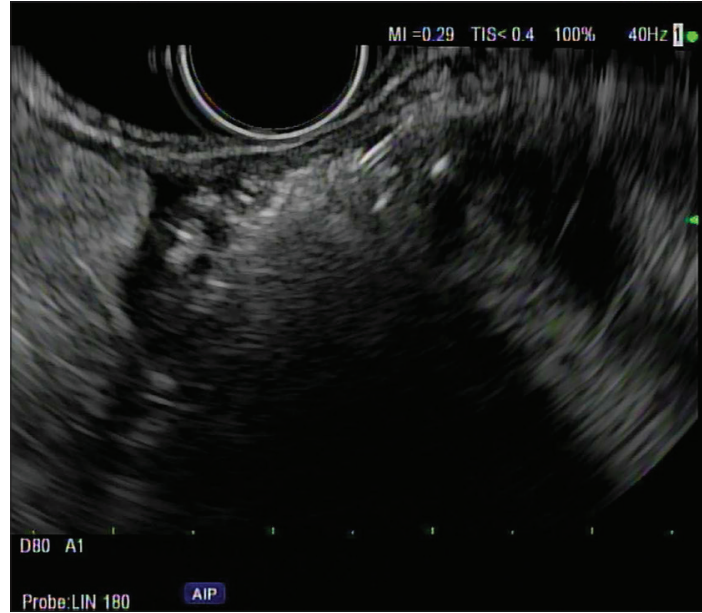

Figure 3. EUS showing an $18 \mathrm{~mm} \times 13 \mathrm{~mm}$ hypoechoic irregular tumor in pancreatic body. The hypoechoic tumor after phosphorus-32 injection is mostly replaced by the hyperechoic "cloud"

Following implantation, it is recommended that patients be observed for $4 \mathrm{~h}$ (with regular observations of consciousness and vital signs as clinically indicated) and if stable and pain-free, they may be discharged the same day.

\section{Assessment of intratumoral localization of} phosphorus-32

Whole-body planar plus single-bed SPECT/CT imaging of P-32 bremsstrahlung radiation is performed within $4 \mathrm{~h}$ postimplantation and again at 7 days, to assess the intratumoral and any potential extratumoral distribution of the P-32 microparticles, as well as, quantify whether or not the entirety of the P-32 radioactivity remained at the implantation site [Figure 4].

The ratio of activity at 7 days to that within $4 \mathrm{~h}$ at the implantation site should be consistent with radioactive decay of P-32 only, indicative of no microparticles having migrated elsewhere.

\section{Treatment follow-up}

The treatment follow-up period consists of investigational visits which occur weekly from visit 1 (Day 0) until week 12, then 4 weeks later at week 16, and then at 8-weekly intervals until study participants reach documented progression of disease criteria for both local progression-free survival and progression-free survival which marks the end of study participation.

Centrally read CT scans are acquired every 8 weeks to assess response defined as complete response, partial response, and stable disease according to Response Evaluation Criteria in Solid Tumor 1.1 criteria.

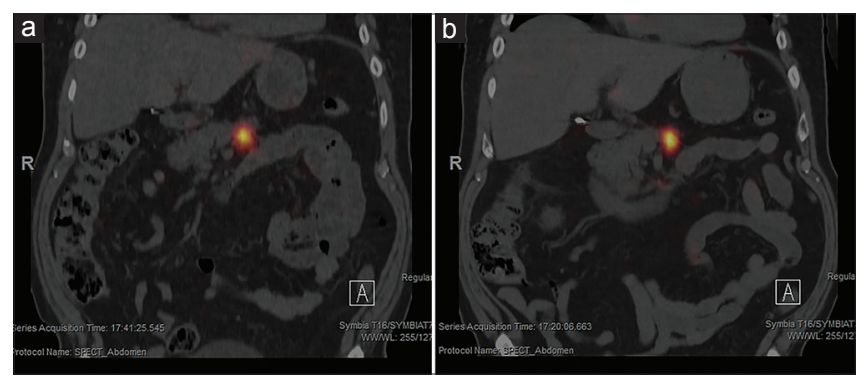

Figure 4. Single photon emission computed tomography-computed tomography bremsstrahlung imaging showing excellent intratumoral retention of phosphorus-32 within the pancreatic body at 4-h postimplantation (a) and at 7-day postimplantation (b)

Telephone contact every 8 weeks is used to monitor device or late radiation-related AEs, and oncology treatments/procedures are administered for up to 12 months post P-32 implantation. Overall survival is assessed by medical record reviews conducted every 8 weeks and/or telephone contact until subject death, or until 104 weeks after the last subject was enrolled.

\section{EUS-GUIDED PHOSPHORUS-32 IMPLANTATION-EARLY RESULTS}

Thus far, a total of 9 patients with a median age of 71 years (range 57-87) have been enrolled in the study at 3 sites in the US. The EUS procedure was technically feasible, and P-32 was successfully implanted in all cases. No serious immediate procedure-related AEs were reported. The clinical trial is ongoing, and additional safety and efficacy data will be presented at the completion of the study.

\section{Financial support and sponsorship}

OncoPac-1 Study is funded by OncoSil Medical Ltd., Sydney, Australia.

\section{Conflicts of interest}

OncoPac-1 Study is funded by OncoSil Medical Ltd., Sydney, Australia. Dr. Bhutani is the recipient of research support from Oncosil, Galera, Augmenix, and Silenseed. Dr. Tamm is associated with General Electric. Dr. Herman is the recipient of research support from Oncosil, Galera, Aduro, and Augmenix, and is a consultant for BTG, AbbVie, Celgene, AstraZeneca, BMS, Varian, and Boston Scientific. The other authors disclosed no financial relationships relevant to this publication.

\section{REFERENCES}

1. Aier I, Semwal R, Sharma A, et al. A systematic assessment of statistics, risk factors, and underlying features involved in pancreatic cancer. Cancer 
Epidemiol 2019;58:104-10.

2. Cardenes HR, Chiorean EG, Dewitt J, et al. Locally advanced pancreatic cancer: Current therapeutic approach. Oncologist 2006;11:612-23.

3. Herman JM, Chang DT, Goodman KA, et al. Phase 2 multi-institutional trial evaluating gemcitabine and stereotactic body radiotherapy for patients with locally advanced unresectable pancreatic adenocarcinoma. Cancer 2015;121:1128-37.

4. Mellon EA, Jin WH, Frakes JM, et al. Predictors and survival for pathologic tumor response grade in borderline resectable and locally advanced pancreatic cancer treated with induction chemotherapy and neoadjuvant stereotactic body radiotherapy. Acta Oncol 2017;56:391-7.

5. Sakurai H, Mitsuhashi N, Harashima K, et al. CT-fluoroscopy guided interstitial brachytherapy with image-based treatment planning for unresectable locally recurrent rectal carcinoma. Brachytherapy 2004;3:222-30.

6. Merrick GS, Butler WM, Wallner KE, et al. Permanent interstitial brachytherapy for clinically organ-confined high-grade prostate cancer with a pretreatment PSA $<20 \mathrm{ng} / \mathrm{mL}$. Am J Clin Oncol 2004;27:611-5.

7. Viola A, Major T, Julow J. The importance of postoperative CT image fusion verification of stereotactic interstitial irradiation for brain tumors. Int J Radiat Oncol Biol Phys 2004;60:322-8.

8. Viswanathan AN, Thomadsen B; American Brachytherapy Society Cervical Cancer Recommendations Committee, American Brachytherapy Society. American Brachytherapy Society consensus guidelines for locally advanced carcinoma of the cervix. Part I: General principles. Brachytherapy 2012;11:33-46.

9. Upcott H. VII. Tumors of the ampulla of vater: With a report of two cases. Ann Surg 1912;56:710-25.

10. Handley WS. Pancreatic cancer and its treatment by implanted radium. Ann Surg 1934;100:215-23.

11. Shipley WU, Nardi GL, Cohen AM, et al. Iodine-125 implant and external beam irradiation in patients with localized pancreatic carcinoma: A comparative study to surgical resection. Cancer 1980;45:709-14.

12. Westlin JE, Andersson-Forsman C, Garske U, et al. Objective responses after fractionated infusional brachytherapy of unresectable pancreatic adenocarcinomas. Cancer 1997;80:2743-8.

13. DeNittis AS, Stambaugh MD, Lang $\mathrm{P}$, et al. Complete remission of nonresectable pancreatic cancer after infusional colloidal phosphorus-32 brachytherapy, external beam radiation therapy, and 5-fluorouracil: A preliminary report. Am J Clin Oncol 1999;22:355-60.

14. Peretz T, Nori D, Hilaris B, et al. Treatment of primary unresectable carcinoma of the pancreas with I-125 implantation. Int J Radiat Oncol Biol Phys 1989;17:931-5.

15. Borgelt B. Radiation therapy with either gold grain implant or neutron beam for unresectable adenocarcinoma of the pancreas. Pancreatic Cancer New Directions in Therapeutic Management. New York: Masson Publishing; 1981. p. 55-62.

16. Order SE, Siegel JA, Principato R, et al. Preliminary experience of infusional brachytherapy using colloidal 32P. Ann Acad Med Singapore 1996;25:347-51.

17. Rosemurgy A, Luzardo G, Cooper J, et al. $32 \mathrm{P}$ as an adjunct to standard therapy for locally advanced unresectable pancreatic cancer: A randomized trial. J Gastrointest Surg 2008;12:682-8.

18. Goertz SR, Ali MM, Parker GA. Local management of pancreatic carcinoma: Iodine-125 implantation. Clin Oncol ( $R$ Coll Radiol) 1990;2:22-6.

19. Du YQ, Li ZS, Jin ZD. Endoscope-assisted brachytherapy for pancreatic cancer: From tumor killing to pain relief and drainage. J Interv Gastroenterol 2011;1:23-7.

20. Cazacu IM, Luzuriaga Chavez AA, et al. A quarter century of EUS-FNA: Progress, milestones, and future directions. Endosc Ultrasound 2018;7:141-60.

21. Ross PJ, Meenan J, O'Doherty M, et al. Novel delivery via endoscopic ultrasound of a 32P brachytherapy device in addition to gemcitabine $(\mathrm{G})$ in advanced pancreatic cancer. Abstract book of ASCO Gastrointestinal Cancers Symposium, January 26-28, American Society for Clinical Oncology's. Abstract no. 205. 